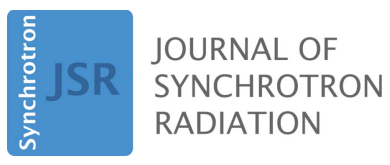

ISSN 1600-5775

Received 24 April 2020

Accepted 10 June 2020

Edited by K. Kvashnina, ESRF - The European Synchrotron, France

Keywords: synchrotron X-ray scanning tunneling microscopy; X-ray chopper;

X-ray absorption spectroscopy.

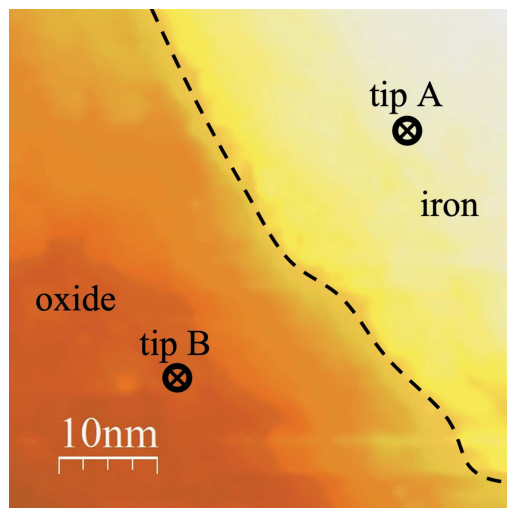

C 2020 International Union of Crystallography

\section{A variable X-ray chopper system for phase-sensitive detection in synchrotron X-ray scanning tunneling microscopy}

\author{
Volker Rose, ${ }^{\mathrm{a}, \mathrm{b} *}$ Tolulope Ajayi, ${ }^{\mathrm{b}}$ Daniel Rosenmann ${ }^{\mathrm{b}}$ and Nozomi Shirato ${ }^{\mathrm{b} *}$ \\ advanced Photon Source, Argonne National Laboratory, 9700 South Cass Avenue, Lemont, IL 60439, USA, and \\ ${ }^{\mathbf{b}}$ Center for Nanoscale Materials, Argonne National Laboratory, 9700 South Cass Avenue, Lemont, IL 60439, USA. \\ *Correspondence e-mail: vrose@anl.gov, nshirato@anl.gov
}

An ultra-high-vacuum compatible X-ray chopper system has been designed, constructed and integrated into the XTIP beamline at the Advanced Photon Source at Argonne National Laboratory. The XTIP beamline can operate at soft $\mathrm{X}$-ray energies from $400 \mathrm{eV}$ to $1900 \mathrm{eV}$ while providing a focused beam down to about $10 \mu \mathrm{m} \times 10 \mu \mathrm{m}$ into the synchrotron X-ray scanning tunneling microscopy (SX-STM) endstation instrument. The X-ray chopper is a critical component for separating topographic information from chemical information in SX-STM through phase-sensitive current detection. Depending on the experimental needs, the modulation frequency can be controlled from $100 \mathrm{~Hz}$ to $10 \mathrm{kHz}$. In addition, the chopper system is fully bakeable and can achieve a base pressure of $10^{-10}$ mbar. Facilities for active water cooling have been designed, but passive cooling through copper braids has been shown to be sufficient at standard chopping frequencies. Using an $\mathrm{Fe} / \mathrm{Al}_{2} \mathrm{O}_{3} / \mathrm{CoAl}(111)$ sample, the separation of the SX-STM current into a chemical component and a stable feedback signal is demonstrated.

\section{Introduction}

X-ray choppers can play a central role in synchrotron beamlines enabling, for example, pump-probe time-resolved measurements (Cammarata et al., 2009; Osawa et al., 2017), laser-pump/X-ray-probe measurements (Graber et al., 2011) or detector characterization (Müller et al., 2015). An X-ray chopper is a further necessary component in synchrotron $\mathrm{X}$-ray scanning tunneling microscopy (SX-STM), where it is required to disentangle sample topography from chemical and magnetic information. In SX-STM, the high spatial resolution of scanning tunneling microscopy (STM) is combined with the electronic and magnetic sensitivity obtained through X-ray/ matter interactions. The technique takes advantage of the fact that X-ray excited electrons can modulate the conventional tunnel current in STM, yielding structural, electronic and magnetic contrast with high spatial resolution (Saito et al., 2006; Chiu, 2008; Okuda et al., 2009; Cummings et al., 2012; Onderwaater et al., 2016). Elemental sensitivity down to the limit of single atomic height (Shirato et al., 2014; Kersell et al., 2017) as well as magnetic contrast (Rose et al., 2012; DiLullo et al., 2016) have been demonstrated. A specialized smart tip serves as a detector for the X-ray enhanced tunneling current (Akiyama et al., 2005; Cummings et al., 2017). The separation between the tip and the sample is typically less than $1 \mathrm{~nm}$ to allow for quantum tunneling and is controlled by an advanced feedback loop (Wang et al., 2013; Mom et al., 2017). However, such a feedback loop requires fast switching between the X-ray beam 'on' and 'off' modes. During the 'off' cycle, the 
tunneling current is governed only by the topography of the sample surface, while the 'on' cycle yields currents that are a convolution of the conventional tunnel current and X-ray excited components. While the tip is raster-scanned over the sample surface, the switching has to be sufficiently fast to allow the feedback loop to continuously adjust the tip height and therewith permit the tip to follow the surface topography. Previously, a different portable chopper system had been designed for SX-STM measurements at other Advanced Photon Source (APS) beamlines (Chang et al., 2016). Here, we report on an advanced X-ray chopper system integrated into XTIP, the first dedicated beamline for the emerging SX-STM technique (Rose et al., 2020). This advanced X-ray chopper system is equipped with temperature sensors and provides cooling capabilities that prevent overheating of the motor. It further includes diagnostics for X-ray beam alignment and characterization.

\section{System design}

\subsection{XTIP beamline}

The XTIP beamline is located at 4-ID of the APS. It features an insertion device that can provide left- or rightcircular as well as horizontal- and vertical-linear polarization. XTIP delivers monochromatic soft X-rays between $400 \mathrm{eV}$ and $1900 \mathrm{eV}$ utilizing a horizontal diffracting spherical-grating monochromator (SGM) (Fig. 1). The SGM focuses the X-ray beam on a movable horizontal exit slit (EXS) with a slit size typically between $10 \mu \mathrm{m}$ and $100 \mu \mathrm{m}$. A focused beam of about $10 \mu \mathrm{m} \times 10 \mu \mathrm{m}$ is then delivered into the SX-STM endstation instrument. In order to reduce the X-ray power load on the chopper, a zero-order slit (ZOS) is used downstream of the SGM.

\subsection{Chopper disk assembly}

Fig. 2 shows the chopper disk assembly. A slotted chopper disk is driven by a customized ultra-high-vacuum (UHV) compatible DC motor (maxon motor). The DC motor is based on an adhesive-free, all pressed and welded construction with a diameter of $26.5 \mathrm{~mm}$. It is equipped with AlNiCo permanent magnets and ironless winding. Since this is a high-temperature

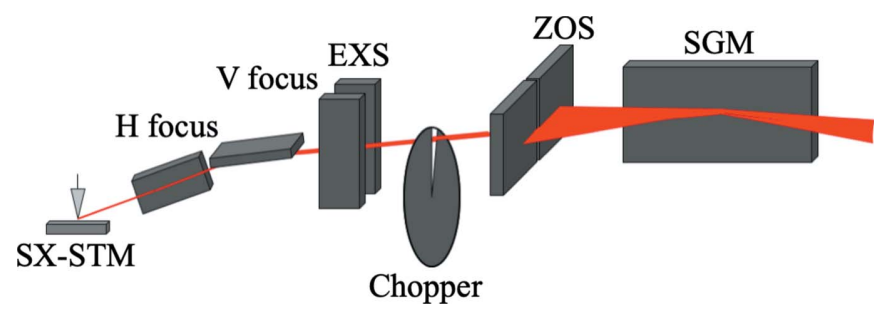

Figure 1

Integration of the X-ray chopper system at the XTIP beamline. A spherical-grating monochromator (SGM) focuses the diffracted X-ray beam onto a moveable exit slit (EXS). The water-cooled zero-order slit (ZOS) removes unwanted diffraction orders and thus reduces the power load on the X-ray chopper. After vertical (V focus) and horizontal (H focus) focusing, the beam illuminates the tip/sample junction in the SXSTM endstation instrument.
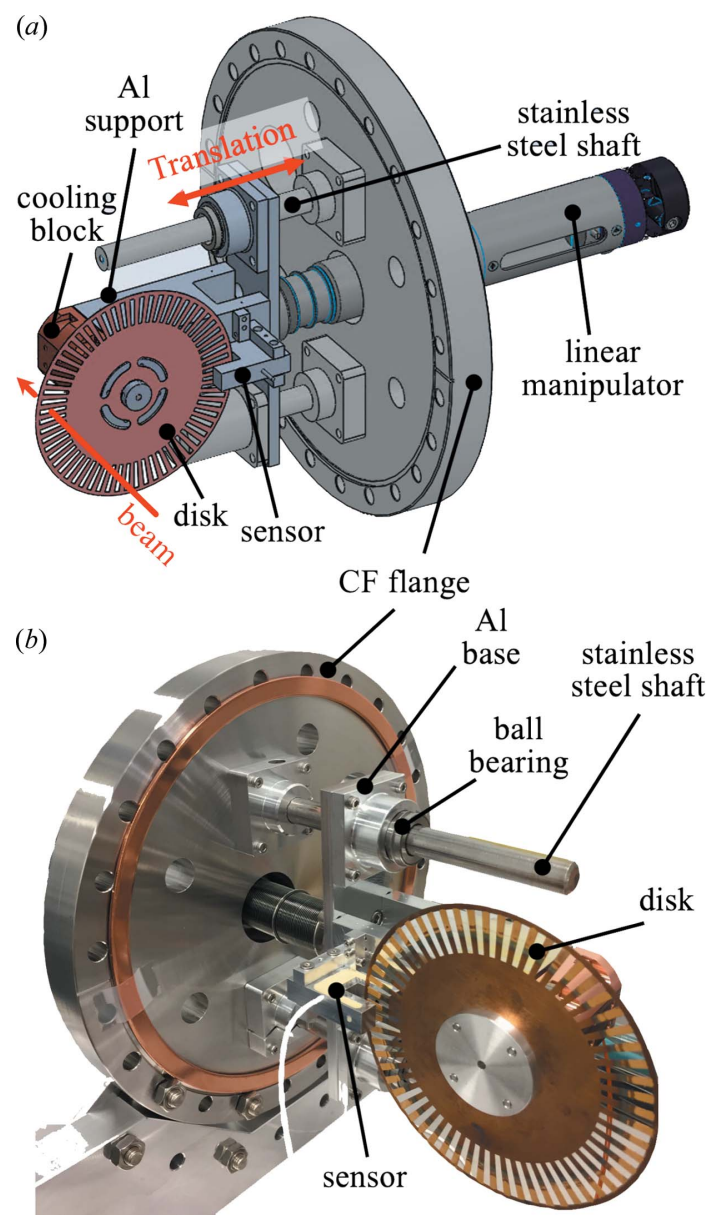

Figure 2

(a) Schematic of the X-ray chopper system. The disk assembly is mounted on a 10 inch $\mathrm{CF}$ flange with a linear translation mechanism used to align the chopper disk to the X-ray beam path. The chopper disk is driven by a motor housed inside a copper cooling block that can be connected to a water-cooling pipe via copper braids (not shown). The chopper frequency is measured by an optical sensor. (b) Photograph of the X-ray chopper system with a 60 -slot disk mounted.

rated motor it can be backed out at a very high temperature $\left(260^{\circ} \mathrm{C}\right)$, limited by the Kapton wire used for the electric connections. The motor is tightly clamped onto a rectangular aluminium support using a gold-plated oxygen-free highly conductive cooling block. The gold plating prevents oxidation during the baking process, which could reduce thermal conductivity. The temperature of the copper cooling block is measured using a silicon diode (Lake Shore). Copper braids are attached to the cooling block in order to dissipate the heat generated by the motor. The water-cooled ZOS located upstream of the chopper system effectively limits the transmitted beam to the first inside order, making the heat load caused by the X-ray beam hitting the chopper disk negligible. A UHV-compatible optical sensor (BOH001J, BALLUFF STM), which is enclosed in an aluminium body, measures the frequency of the chopper. A linear translation mechanism mounted on the 10 inch ConFlat (CF) flange allows for the alignment of the chopper disk with respect to the X-ray beam path. Self-aligning ball-bearing units, which are attached to the 

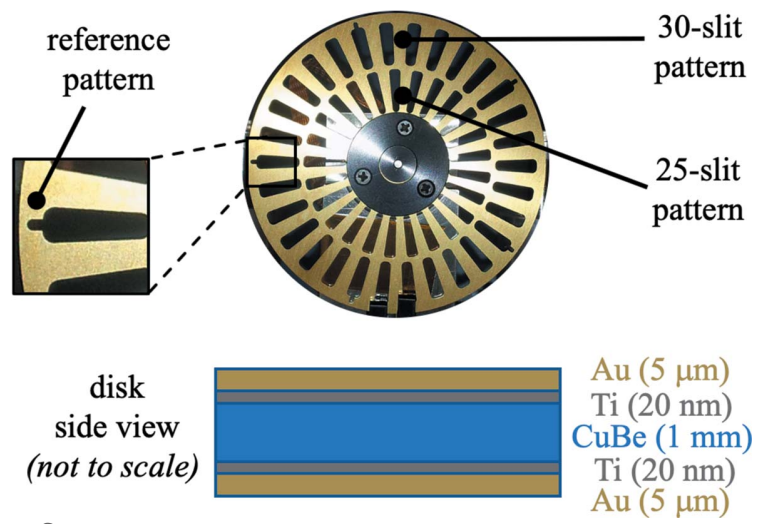

Figure 3

Photograph of a chopper disk featuring 30- and 25-slit patterns. The reference pattern on the outside of the disk is located at the position detected by the optical sensor. All disks for the chopper assembly are machined from a $\mathrm{CuBe}$ alloy and subsequently coated by electron-beam evaporation with a $20 \mathrm{~nm}$-thick titanium seed layer and a $5 \mu \mathrm{m}$ gold film on each side of the disk.

top and bottom of the aluminium base, facilitate a linear translation of the base and the chopper disk guided by two stainless steel shafts. The ball-bearing units provide a rigid and low drag linear movement. A commercial optical chopper controller (model 3502, New Focus) is used to power the DC motor and maintain a stable chopping frequency that is phaselocked to the reference signal provided by the optical sensor.

In addition to the 60-slot pattern shown in Fig. 2, disks (115 mm diameter, $1 \mathrm{~mm}$-thick) with various slot patterns are available, providing modulation frequencies from $100 \mathrm{~Hz}$ to $10 \mathrm{kHz}$. Fig. 3 shows a disk with a 25- and 30-slit pattern. An additional reference pattern is located on the outside of the disk at a position detected by the optical sensor when the disk is mounted in the chopper assembly. This provides the reference signal for phase-sensitive analysis of signals. All slotted chopper disks are machined of a $\mathrm{CuBe}$ alloy and then coated by electron-beam evaporation with a $20 \mathrm{~nm}$-thick Ti seed layer and a $5 \mu \mathrm{m}$-thick $\mathrm{Au}$ film on each side of the disk to improve the surface finish and X-ray absorption.

\subsection{Chopper system}

The chopper disk assembly is mounted on a dedicated UHV chamber that has been integrated into the XTIP beamline (see Fig. 1). Fig. 4 shows a rendering of the UHV chamber housing the chopper disk assembly. The chamber is mounted on a support stand that facilitates height alignment of the chopper system. The fine adjustment of the chopper disk is achieved using a linear manipulator. In order to maintain UHV conditions the chopper system includes a non-evaporable getter (NEG) and ion combination pump. A linear transfer downstream of the chopper disk provides the option to move diagnostics such as a YAG crystal or photodiode into the beam to aid the alignment procedure of the chopper disk. The chamber further exhibits a port for optional water cooling of the DC motor. Utilizing flexible vacuum bellows between the chopper system and the beamline reduces the potential transmission of vibrational noise from the chopper system into
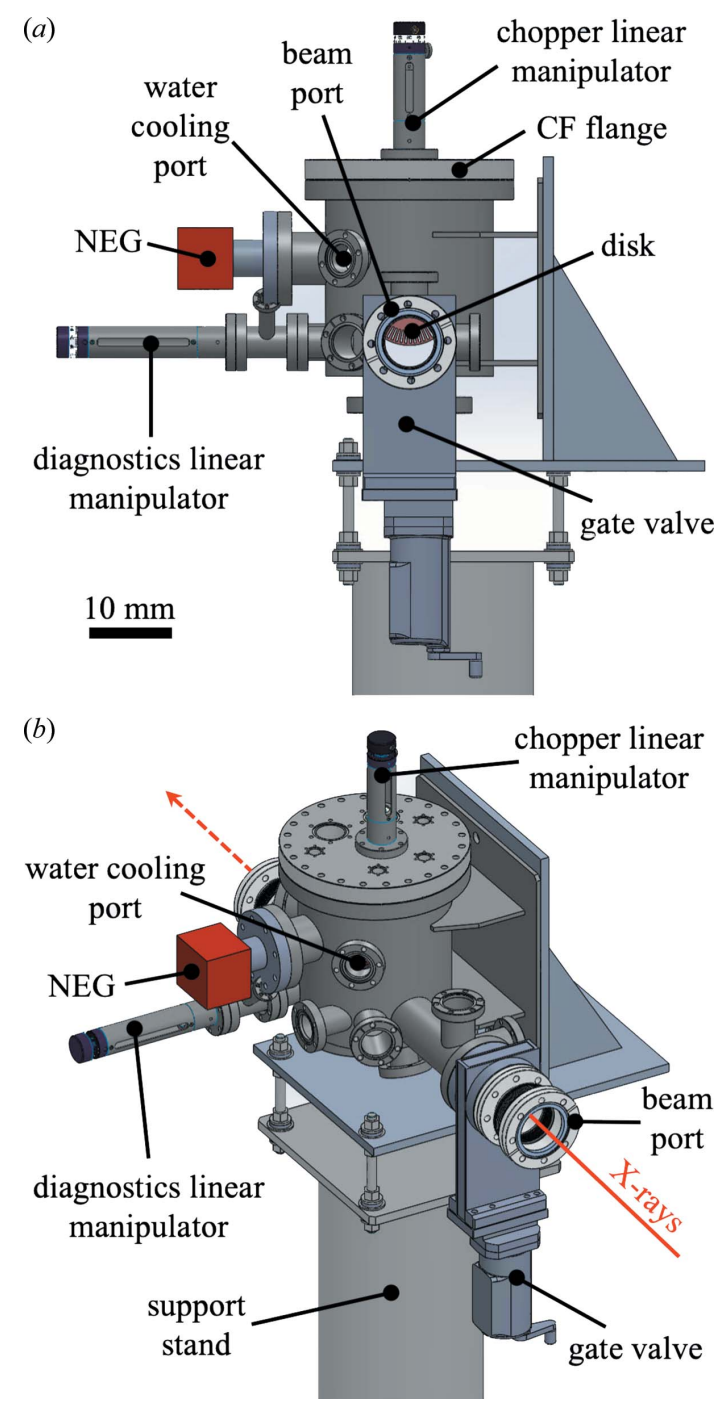

Figure 4

Schematic of the UHV chamber housing the chopper assembly. The chamber features an NEG and ion combination pump as well as a linear transfer to bring diagnostics into the beam path downstream of the chopper disk. (a) Downstream view. (b) Rendering showing the X-ray beam path.

the SX-STM endstation instrument. After baking, the chopper system achieves a base pressure of $10^{-10} \mathrm{mbar}$. The pressure is preserved during operation of the chopper.

\section{Results}

\subsection{Operating temperature}

Heat generated by a moving part under UHV conditions often imposes significant challenges. This is particularly true for a continuously moving DC motor that has a limited range of allowable temperatures for operation. Therefore, the motor driving the chopper disk was connected to a heat sink via copper braids. Additionally, the UHV chamber provides the option for water cooling, but, as shown here, under standard operating frequencies, passive cooling through the copper braids is satisfactory. Fig. 5 shows the temperature measured 


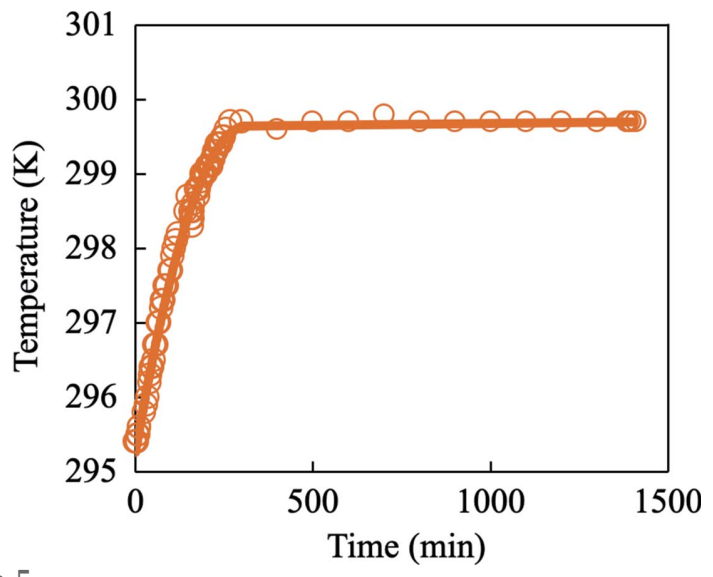

Figure 5

Temperature of the chopper assembly measured at the copper cooling block while operating for $24 \mathrm{~h}$ at a chopping frequency of $2.8 \mathrm{kHz}$.

at the motor cooling block over a $24 \mathrm{~h}$ period of continuous operation of the chopper. The motor load corresponds to a chopping frequency of $2.8 \mathrm{kHz}$. During the initial $300 \mathrm{~min}$ of operation the temperature increases from $295.5 \mathrm{~K}$ to $299.7 \mathrm{~K}$. Afterwards, an equilibrium is reached and the temperature remains stable below $300 \mathrm{~K}$. These are suitable conditions for long-term operation of the chopper. Therefore, additional water cooling is not required.

\subsection{Disentangling topography and spectroscopy}

An X-ray chopper is a necessary component in SX-STM because the current measured at the tip detector is a convolution of the conventional tunnel current (related to sample topography) and X-ray excited electrons (associated with chemical and magnetic properties). Without the use of a chopper system that enables an advanced feedback loop, $\mathrm{X}$-rays can cause a transition of the tip out of the tunneling regime (Rose et al., 2013). Therefore, the chopper system has the objective of providing a stable tip/sample separation that is only governed by the sample topography and not by X-ray excited electrons. Fig. 6(a) shows the two conditions offered by a chopper. When the X-ray chopper is blocking the beam, only the conventional tunnel current is present. This condition is the same as that of a conventional STM. The tunnel current can then be used for the feedback loop. On the other hand, when the X-rays pass through the chopper disk and illuminate the tip/sample junction, an X-ray excited current is generated in addition to the conventional tunnel current. Since the current signal now carries a convolution of topographic and chemical information, it cannot directly be used for the feedback loop. Therefore, the time sequence of the SX-STM current signal [Fig. 6(b)] has to be analyzed to enable a continuous feedback. The reference slit pattern in the disk that is measured by the optical sensor yields the chopper reference signal. It defines the 'on' and 'off' cycle of the chopper. The phase-sensitive detection of the current is then used to obtain a suitable signal for the feedback. During the 'off' cycle, only the conventional tunnel current is measured and can be directly used as a feedback signal. This signal is

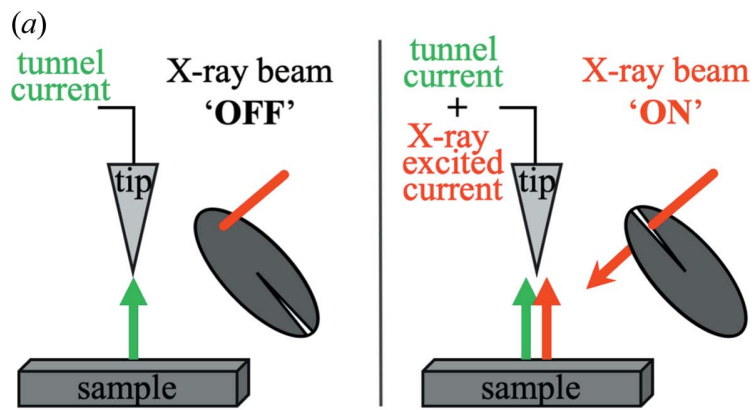

(b)
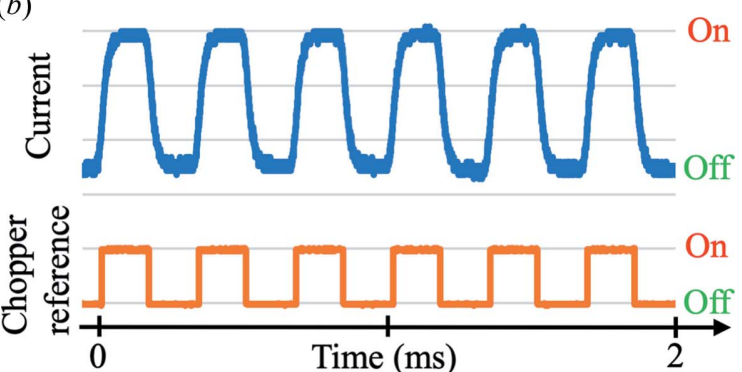

Figure 6

(a) When the chopper is blocking the X-ray beam ('off'), only the conventional tunneling current is present. When the X-ray beam passes through the chopper ('on'), an additional X-ray excited current is generated. (b) Time sequence of the SX-STM current signal (top) and the associated chopper reference (bottom) recorded with the tip detector tunneling over the sample.

then also supplied to the feedback during the following 'on' cycle. This continuous sequence of measuring and holding, resulting from real-time signal processing in a field-programmable gate array (FPGA), now yields a proper feedback signal. Interestingly, in addition to obtaining the feedback signal, phase-sensitive analysis of the current can also deliver chemical contrast. This is achieved by analysing the current during the 'on' cycles in which X-ray excited current is produced on top of the conventional tunnel current. Thus, the chopper system in conjunction with phase-sensitive analysis of the current allows for the separation of topographic and chemical information while allowing their simultaneous measurement. Additionally, magnetic information becomes available when polarized X-rays are used.

In order to verify the functionality of the chopper system for SX-STM measurements, a $\mathrm{CoAl}(111)$ single crystal was cleaned by several cycles of $\mathrm{Ar}^{+}$sputtering and annealing. Subsequently, the sample was oxidized with $500 \mathrm{~L} \mathrm{O}_{2}$ at $800 \mathrm{~K}$ $\left(1 \mathrm{~L}=1.3 \times 10^{-8} \mathrm{mbar}\right)$. It is known that the intermetallic alloy $\mathrm{CoAl}$ forms ultra-thin alumina films with a self-limiting thickness of about $1 \mathrm{~nm}$, thin enough to still allow for electron tunneling through the oxide (Rose et al., 2005). After oxidation, a sub-monolayer of $\mathrm{Fe}$ is deposited on the surface. Fig. 7 shows a topography scan of the $\mathrm{Fe} / \mathrm{Al}_{2} \mathrm{O}_{3} / \mathrm{CoAl}(111)$ sample surface. A metal/insulator/metal smart tip (with a tungsten core, silicon dioxide insulating layer and gold conductive outer shield) is used for the measurements.

Subsequently, point spectra are obtained at different sample positions, represented by locations labeled 'tip A' and 'tip B'. 


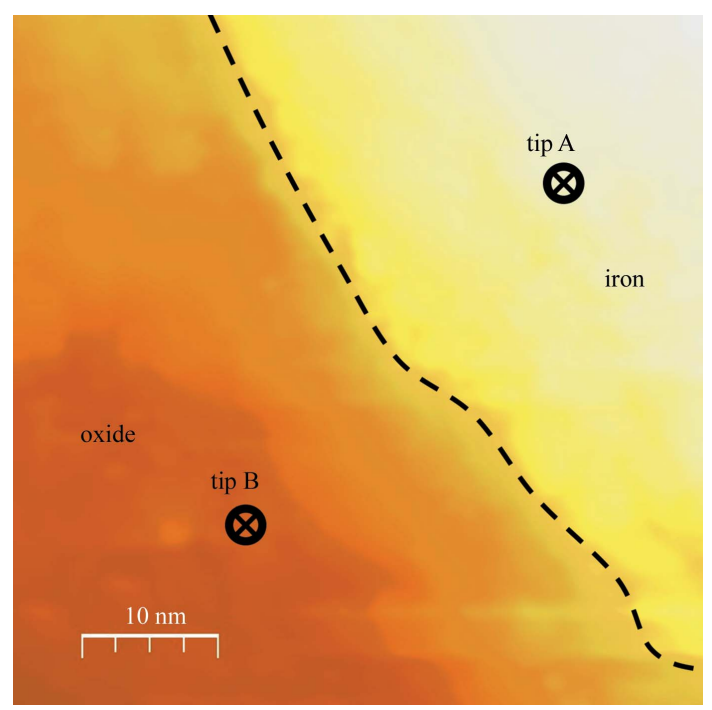

Figure 7

Topographic scan after the deposition of a sub-monolayer Fe film on an oxidized CoAl single crystal. Two locations (tip A and tip B) are indicated and used for point spectroscopy measurements. The tunneling current setpoint is $0.1 \mathrm{nA}$ and the bias voltage is $-1 \mathrm{~V}$.

To acquire a point spectrum, the lateral tip position is fixed and the tip height is maintained by the feedback loop in constant current mode with a setpoint of $0.1 \mathrm{nA}$. Then the $\mathrm{X}$-ray photon energy is ramped over the absorption edge of interest. Fig. 8( $a)$ shows the spectra acquired at the location represented by 'tip A', where the currents during the 'on' and 'off' cycles are measured simultaneously. During the 'off' cycle a constant current of $0.1 \mathrm{nA}$ is maintained. This demonstrates that the signal for the feedback loop is not impacted by the $\mathrm{X}$-ray illumination and the topographic information can be successfully extracted. At the same time, the phase-sensitive analysis of the 'on' cycle yields the chemical information at the location 'tip A'. The spectrum exhibits the $\mathrm{Fe} L_{3}$ and $\mathrm{Fe} L_{2}$ absorption peaks at $707 \mathrm{eV}$ and $720 \mathrm{eV}$, respectively. This suggests the presence of an $\mathrm{Fe}$ island. In contrast, the absorption spectrum at location 'tip B' presents the oxygen $K$ absorption edge at about $540 \mathrm{eV}$ [Fig. 8(b)]. Thus, location 'tip B' suggests the presence of the aluminium oxide film that is not covered by iron. Like in the case of location 'tip A', the 'off' cycle yields a tunneling current setpoint of $0.1 \mathrm{nA}$. This proves that the signal for the feedback loop is successfully extracted at different absorption edges and with both high and low absorption intensity. Hence, the chopper system allows us to disentangle the topographic information from the chemical information while obtaining both parameters simultaneously.

\section{Conclusions}

An X-ray chopper system with a base pressure of $10^{-10} \mathrm{mbar}$ has been developed and integrated into the XTIP beamline for modulation frequencies from $100 \mathrm{~Hz}$ to $10 \mathrm{kHz}$. Lightweight chopper disks with high stiffness have been manufactured that can block soft and hard X-rays. Although the motor driving the chopper disk can be water-cooled, the use
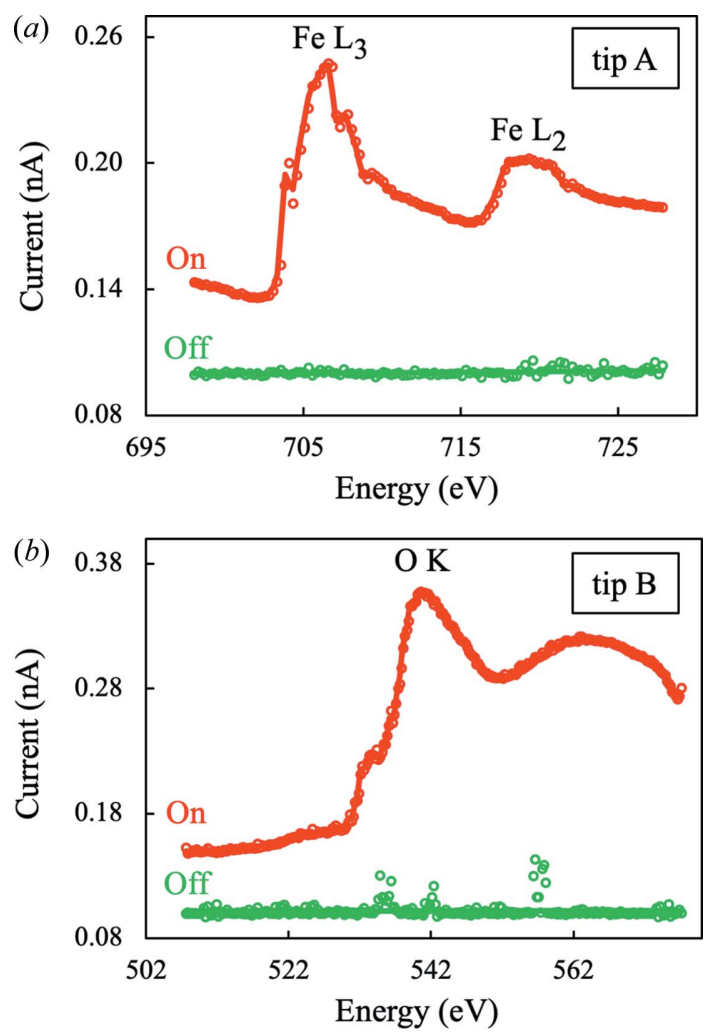

Figure 8

X-ray absorption spectra of the $\mathrm{Fe} / \mathrm{Al}_{2} \mathrm{O}_{3} / \mathrm{CoAl}(111)$ sample using phasesensitive current detection of the 'on' and 'off' cycles of the chopper system. (a) At location 'tip A' the spectrum with X-ray illumination ('on') exhibits $\mathrm{Fe} L_{3}$ and $\mathrm{Fe} L_{2}$ absorption edges, indicating the presence of an Fe island. During the photon energy ramp, the tunneling current setpoint, i.e. the feedback signal of $0.1 \mathrm{nA}$, is maintained as demonstrated by the phase-sensitive detection of the 'off' cycle. (b) Similarly, at location 'tip B' the chemical fingerprint of the oxide film can be observed, while the tip detector maintains stable tunneling conditions at $0.1 \mathrm{nA}$.

of passive cooling through copper braids has proven to be sufficient at chopping frequencies that represent standard operational conditions for SX-STM. The chopper system is a crucial component for SX-STM measurements. Using an Fe/ $\mathrm{Al}_{2} \mathrm{O}_{3} / \mathrm{CoAl}(111)$ sample grown in situ the chopper system has enabled the separation of topographic and chemical information in SX-STM. In addition to facilitating SX-STM measurements, UHV-capable chopper systems have the potential to enable a wide variety of X-ray techniques. For example, the implementation of an identical chopper system as part of the APS upgrade is currently under consideration for the Polarization Modulation Spectroscopy Beamline.

\section{Acknowledgements}

The authors thank Alex Deriy for his help with the installation of the chopper system at XTIP.

\section{Funding information}

This work was performed at the Advanced Photon Source and the Center for Nanoscale Materials, US Department of Energy Office of Science User Facilities, and supported by the 
US Department of Energy, Office of Science (contract No. DE-AC02-06CH11357).

\section{References}

Akiyama, K., Eguchi, T., An, T., Hasegawa, Y., Okuda, T., Harasawa, A. \& Kinoshita, T. (2005). Rev. Sci. Instrum. 76, 083711.

Cammarata, M., Eybert, L., Ewald, F., Reichenbach, W., Wulff, M., Anfinrud, P., Schotte, F., Plech, A., Kong, Q., Lorenc, M., Lindenau, B., Räbiger, J. \& Polachowski, S. (2009). Rev. Sci. Instrum. 80, 015101.

Chang, H., Cummings, M., Shirato, N., Stripe, B., Rosenmann, D., Preissner, C., Freeland, J. W., Kersell, H., Hla, S.-W. \& Rose, V. (2016). AIP Conf. Proc. 1696, 020001.

Chiu, C.-Y., Chan, Y.-L., Hsu, Y. J. \& Wei, D. H. (2008). Appl. Phys. Lett. 92, 103101.

Cummings, M., Shirato, N., Kersell, H., Chang, H., Rosenmann, D., Freeland, J. W., Miller, D., Hla, S. W. \& Rose, V. (2017). J. Appl. Phys. 121, 015305.

Cummings, M. L., Chien, T. Y., Preissner, C., Madhavan, V., Diesing, D., Bode, M., Freeland, J. W. \& Rose, V. (2012). Ultramicroscopy, 112, 22-31.

DiLullo, A., Shirato, N., Cummings, M., Kersell, H., Chang, H., Rosenmann, D., Miller, D., Freeland, J. W., Hla, S.-W. \& Rose, V. (2016). J. Synchrotron Rad. 23, 574-578.

Graber, T., Anderson, S., Brewer, H., Chen, Y.-S., Cho, H. S., Dashdorj, N., Henning, R. W., Kosheleva, I., Macha, G., Meron, M., Pahl, R., Ren, Z., Ruan, S., Schotte, F., Šrajer, V., Viccaro, P. J., Westferro, F., Anfinrud, P. \& Moffat, K. (2011). J. Synchrotron Rad. 18, 658-670.

Kersell, H., Shirato, N., Cummings, M., Chang, H., Miller, D., Rosenmann, D., Hla, S.-W. \& Rose, V. (2017). Appl. Phys. Lett. 111, 103102.
Mom, R. V., Onderwaater, W. G., Rost, M. J., Jankowski, M., Wenzel, S., Jacobse, L., Alkemade, P. F. A., Vandalon, V., van Spronsen, M. A., van Weeren, M., Crama, B., van der Tuijn, P., Felici, R., Kessels, W. M. M., Carlà, F., Frenken, J. W. M. \& Groot, I. M. N. (2017). Ultramicroscopy, 182, 233-242.

Müller, O., Lützenkirchen-Hecht, D. \& Frahm, R. (2015). Rev. Sci. Instrum. 86, 035105.

Okuda, T., Eguchi, T., Akiyama, K., Harasawa, A., Kinoshita, T., Hasegawa, Y., Kawamori, M., Haruyama, Y. \& Matsui, S. (2009). Phys. Rev. Lett. 102, 105503.

Onderwaater, W. G., van der Tuijn, P. C., Mom, R. V., van Spronsen, M. A., Roobol, S. B., Saedi, A., Drnec, J., Isern, H., Carla, F., Dufrane, T., Koehler, R., Crama, B., Groot, I. M. N., Felici, R. \& Frenken, J. W. M. (2016). Rev. Sci. Instrum. 87, 113705.

Osawa, H., Kudo, T. \& Kimura, S. (2017). Jpn. J. Appl. Phys. 56, 048001.

Rose, V., Chien, T. Y., Freeland, J. W., Rosenmann, D., Hiller, J. \& Metlushko, V. (2012). J. Appl. Phys. 111, 07E304.

Rose, V., Podgursky, V., Costina, I., Franchy, R. \& Ibach, H. (2005). Surf. Sci. 577, 139-150.

Rose, V., Shirato, N., Bartlein, M., Deriy, A., Ajayi, T., Rosenmann, D., Hla, S.-W., Fisher, M. \& Reininger, R. (2020). J. Synchrotron Rad. 27, 836-843.

Rose, V., Wang, K., Chien, T. Y., Hiller, J., Rosenmann, D., Freeland, J. W., Preissner, C. \& Hla, S.-W. (2013). Adv. Funct. Mater. 23, 26462652.

Saito, A., Maruyama, J., Manabe, K., Kitamoto, K., Takahashi, K., Takami, K., Yabashi, M., Tanaka, Y., Miwa, D., Ishii, M., Takagi, Y., Akai-Kasaya, M., Shin, S., Ishikawa, T., Kuwahara, Y. \& Aono, M. (2006). J. Synchrotron Rad. 13, 216-220.

Shirato, N., Cummings, M., Kersell, H., Li, Y., Stripe, B., Rosenmann, D., Hla, S.-W. \& Rose, V. (2014). Nano Lett. 14, 6499-6504.

Wang, K., Rosenmann, D., Holt, M., Winarski, R., Hla, S.-W. \& Rose, V. (2013). Rev. Sci. Instrum. 84, 063704. 